\title{
Experimental realization of the classical Dicke model
}

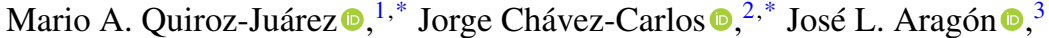 \\ Jorge G. Hirsch ${ }^{1, \dagger},{ }^{\dagger}$ and Roberto de J. León-Montiel $\oplus^{1, \dagger}$ \\ ${ }^{1}$ Instituto de Ciencias Nucleares, Universidad Nacional Autónoma de México, Apartado Postal 70-543, 04510 CDMX, México \\ ${ }^{2}$ Instituto de Ciencias Físicas, Universidad Nacional Autónoma de México, Av. Universidad s/n Col. Chamilpa, C.P. 62210 Cuernavaca, \\ Morelos, México \\ ${ }^{3}$ Centro de Física Aplicada y Tecnología Avanzada, Universidad Nacional Autónoma de México, Boulevard Juriquilla 3001, \\ 76230 Querétaro, México
}

(Received 28 January 2020; revised 14 May 2020; accepted 14 July 2020; published 30 July 2020)

\begin{abstract}
We report the experimental implementation of the classical description of the Dicke model whose quantum version describes a large number of two-level atoms interacting with a single-mode electromagnetic field in a perfectly reflecting cavity. This is performed by employing two nonlinearly coupled active, synthetic LC circuits, implemented by means of analog electrical components. The simplicity and versatility of our platform allows us not only to experimentally explore the coexistence of regular and chaotic trajectories in the classical Dicke model, but also to directly observe the so-called ground-state and excited-state "quantum" phase transitions. In this analysis, the trajectories in phase space, Lyapunov exponents, and the finite-time Lyapunov exponent are used to identify the different operating regimes of our electronic device. Moreover, with this technology, we measure the classic analog of the fidelity-out-of-time-order-correlator (FOTOC). Exhaustive numerical simulations are performed to show the quantitative and qualitative agreement between theory and experiment.
\end{abstract}

DOI: 10.1103/PhysRevResearch.2.033169

\section{INTRODUCTION}

The quantum dynamics of isolated many-body systems can reach states of equilibrium, in some cases allowing for a thermal description, when they are in a chaotic regime [1-11]. While equilibrium is associated with small fluctuations around an average value of some observables whose relative ratio becomes smaller as the size of the system is increased, thermalization refers to the agreement of these average values with those obtained by means of statistical mechanics. Determining under which conditions a given quantum system equilibrates is both relevant and challenging. Interestingly, chaos plays a fundamental role in the equilibration of a quantum system under unitary dynamics $[12,13]$. It makes particularly important the study of quantum systems whose classical counterparts are chaotic [14-16], a very common situation as the classical dynamics of many-body systems in interaction is in most cases nonlinear, nonintegrable, chaotic, as they have less integrals of motion than degrees of freedom.

Originally introduced as the quantum description of the collective behavior of two-level atoms interacting with a single electromagnetic mode of a cavity, the Dicke model $[17,18]$ has found application in the study of equilibrium

\footnotetext{
${ }^{*}$ These authors contributed equally to this work.

†hirsch@nucleares.unam.mx

†roberto.leon@nucleares.unam.mx
}

Published by the American Physical Society under the terms of the Creative Commons Attribution 4.0 International license. Further distribution of this work must maintain attribution to the author(s) and the published article's title, journal citation, and DOI. and thermalization of isolated quantum many-body systems, as well as their classical-quantum correspondence [19-28]. Recent experiments with ion traps have shed new light on the system [29,30], and have opened the possibility of observing quantum indicators of chaos, such as the fidelity-out-of-timeorder-correlator (FOTOC), at short times in the Dicke model [31].

The Dicke model has been a subject of intensive research for many years. In particular, it has been shown to exhibit quantum phase transitions in the ground [32-34] and the excited states [35-39]. Indications of the presence of quantum chaos in the fluctuation of its energy spectra were reported in [33] and studied in detail in [40]. The presence of regular and chaotic regions has been analyzed qualitatively employing Poincaré sections for the classical analysis and Peres lattices in the quantum case [41], followed by a detailed quantitative analysis of the classical phase space and the parameter space performed by means of Lyapunov exponents [42]. Along these lines, it has been proven that there exists a clear correlation between the Lyapunov exponents and the participation ratio of the coherent states in the Dicke Hamiltonian eigenbasis [43]. Furthermore, the out-of-time-order-correlator (OTOC) has been shown to grow at short times with a ratio close to the Lyapunov exponent [44].

The classical Dicke model has played a fundamental role in understanding the emergence of symmetric states, important phase transitions, and the existence of superradiance in the ground state [32-37]. Surprisingly, an aspect which has never before been shown is its physical realization. In this work, we present its first implementation. This is done by making use of two synthetic, nonlinearly coupled electrical LC (where L stands for inductance and C for capacitance) oscillators, implemented by means of analog electrical 
components. Our platform is thus used to observe the dynamics of the classical Dicke model and monitor important features, such as the ground-state and excited-state phase transitions, the coexistence of periodic and chaotic trajectories, and the classical version of the FOTOC. Because of its simplicity and versatility, our platform constitutes an excellent test bed for exploring the richness of nonlinear systems in terms of chaos and regularity.

The paper is structured as follows. In Sec. II, we present the semiclassical approximation of the Dicke model and the Hamiltonian describing its classical dynamics. In Sec. III, we express the classical Dicke Hamiltonian in terms of the electrical variables of LC oscillators. In Sec. IV, we describe the dynamical evolution of the system and discuss some representative phenomena of the classical Dicke model and finally, in Sec. V, we present our conclusions.

\section{CLASSICAL DESCRIPTION OF THE DICKE HAMILTONIAN}

In this section, we briefly describe the classical approximation of the Dicke model and its corresponding Hamiltonian. We start by considering the quantum Hamiltonian for $N$ identical two-level atoms under the action of a single-mode quantized radiation field (Dicke model) [17], which reads $(\hbar=1)$

$$
H_{D}=\omega \hat{a}^{\dagger} \hat{a}+\omega_{0} \hat{J}_{z}+\frac{\gamma}{\sqrt{N}}\left(\hat{J}_{+}+\hat{J}_{-}\right)\left(\hat{a}^{\dagger}+\hat{a}\right),
$$

where $\omega$ is the frequency of the monochromatic quantized radiation field and $\omega_{0}$ is the excitation energy of a set of two-level atoms. $\hat{a}$ and $\hat{a}^{\dagger}$ represent the one-mode annihilation and creation photon operators, respectively. $\hat{J}_{z}, \hat{J}_{+}$, and $\hat{J}_{-}$are collective atomic pseudospin operators which obey the $S U(2)$ algebra. Specifically, $\hat{J}_{z}$ is the atomic relative population operator and $\hat{J}_{ \pm}$are the atomic transition operators. If $j(j+1)$ is an eigenvalue of angular momentum operator $\hat{J}^{2}$, then $j=$ $N / 2$ defines the symmetric atomic subspace, including the ground state. Finally, $\gamma$ is the matter-light coupling strength, which depends on the atomic dipolar moment. When the coupling strength reaches the critical value $\gamma_{c}=\sqrt{\omega \omega_{0}} / 2$, the ground state of the system goes from a normal to a superradiant phase. This behavior is the most representative phenomena of the Dicke Hamiltonian in the thermodynamic limit: the quantum phase transition (QPT) [33]. Another important feature of the Dicke Hamiltonian is the presence of an excited state quantum phase transition (ESQPT) manifested by simultaneous singularities in the eigenvalue spectrum, order parameters, and wave-function properties $[36,45,46]$.

By employing Glauber coherent states given by $|\alpha\rangle=$ $e^{-|\alpha|^{2} / 2} e^{\alpha \hat{a}^{\dagger}}|0\rangle$ and Bloch coherent states described by $|z\rangle=$ $1 /\left(1+|z|^{2}\right)^{-j} e^{z \hat{J}_{+}}|j,-j\rangle$, one can find an effective classical Hamiltonian by taking the expectation value of the Hamiltonian operator in the coherent state product [47]. The classical Hamiltonian per particle, $\left\langle\alpha, z\left|H_{D}\right| \alpha, z\right\rangle / j$, in terms of the canonical variables $(p, q)$ and $(P, Q)$, reads $[42,43]$

$H_{c l}=\frac{\omega}{2}\left(p^{2}+q^{2}\right)+\frac{\omega_{0}}{2}\left(P^{2}+Q^{2}\right)+\gamma q Q \sqrt{4-P^{2}-Q^{2}}-\omega_{0}$, where $(p, q)$ and $(P, Q)$ are pairs of real conjugate coordinates for the photonic and the atomic sectors, respectively. They are given in terms of the coherent state parameters as

$$
\begin{gathered}
\alpha=\sqrt{\frac{j}{2}}(q+i p), \\
z=\frac{Q-i P}{\sqrt{4-\left(Q^{2}+P^{2}\right)}} .
\end{gathered}
$$

The dynamical properties of the Hamiltonian (2) can be described by the temporal evolution of the canonical variables $\mathbb{X}=(p, q, P, Q)$ whose equations of motion are [42]

$$
\begin{aligned}
& \dot{p}=-\gamma Q \sqrt{4-P^{2}-Q^{2}}-q \omega, \\
& \dot{q}=p \omega, \\
& \dot{P}=\frac{\gamma q Q^{2}}{\sqrt{4-P^{2}-Q^{2}}}-\gamma q \sqrt{4-P^{2}-Q^{2}}-Q \omega_{0}, \\
& \dot{Q}=P \omega_{0}-\frac{\gamma P q Q}{\sqrt{4-P^{2}-Q^{2}}} .
\end{aligned}
$$

Note that the field variables $(p, q)$ are unbound, while the atomic variables $(P, Q)$ must satisfy the condition $P^{2}+Q^{2} \leqslant$ 4 , reflecting the fact that the quantum angular momentum space employed to describe the collective atomic excitations is finite, with dimension $2 j+1$. In the classical dynamics governed by Hamiltonian (2) there is no reference to $j$, this inequality is a memory of it in the classical realm, with the point $P=Q=0$ associated with the South pole of the Bloch sphere, and the circumference defined by $P^{2}+Q^{2}=4$ with the North pole.

The equilibrium points can be easily determined by equating the dynamical equations (5) to zero, $\mathbf{F}(\mathbb{X})=\overline{0}$, and simultaneously solving the set of algebraic equations. In doing so, we obtain the following critical points:

$$
\begin{aligned}
& \mathfrak{X}_{\mathbb{D}}=(0,0,0,0), \\
& \mathfrak{X}_{ \pm}=\left(0, \pm \frac{2 \gamma^{2}}{\omega} \sqrt{1-\frac{\gamma_{c}^{4}}{\gamma^{4}}}, 0, \mp \sqrt{2\left(1-\frac{\gamma_{c}^{2}}{\gamma^{2}}\right)}\right),
\end{aligned}
$$

Note that if $\gamma>\gamma_{c}$, the system has three real equilibrium points, and when $\gamma \leqslant \gamma_{c}, \mathbb{K}_{0}$ is the only real equilibrium point. This result is consistent with the so-called saddle-node bifurcation, characterized by the annihilation of two points of equilibrium in a dynamical system [48]. Next, we consider the dynamics of the system in the neighborhood of $\mathbb{X}_{0}$. The Jacobian matrix $\mathbb{l}=D_{\mathbb{K}} \mathbf{F}(\mathbb{X})$ evaluated at this point is given by

$$
\rrbracket^{\mathbb{X}_{\mathbb{O}}}=\left(\begin{array}{cccc}
0 & -\omega & 0 & -2 \gamma \\
\omega & 0 & 0 & 0 \\
0 & -2 \gamma & 0 & -\omega_{0} \\
0 & 0 & \omega_{0} & 0
\end{array}\right),
$$

and its eigenvalues, in the resonant case $\omega=\omega_{0}$, are described by $\lambda\left(\mathbb{J}^{\mathcal{X}_{0}}\right)=\left\{\mp 2 \gamma_{c} \sqrt{\frac{\gamma}{\gamma_{c}}-1}, \mp 2 i \gamma_{c} \sqrt{\frac{\gamma}{\gamma_{c}}+1}\right\}$. It follows from the above that Eq. (7) has two real and two purely imaginary eigenvalues (hyperbolic point) when the interaction strength $\gamma>\gamma_{c}$ and become purely imaginary (center point) when the interaction strength is smaller, i.e., $\gamma<\gamma_{c}$. This stability 
change signals the so-called Andronov-Hopf bifurcation, in which an equilibrium switches its stability via a pair of purely imaginary eigenvalues [48,49]. By making a similar analysis in the equilibrium points $\mathbb{K}_{ \pm}$, one can find that they correspond to center points. Notably, for $\gamma=\gamma_{c}$ and $E=-1$, both center points collide with the hyperbolic point, giving rise to a phenomenon that resembles a homoclinic bifurcation $[38,40,45,48]$.

At the transition point $\gamma=\gamma_{c}$ and $E=-1, \mathbb{X}_{0}$ undergoes a Bogdanov-Takens (BT) bifurcation [48,50], which meets the three codimension-one bifurcations: saddle-node bifurcation, Andronov-Hopf bifurcation, and homoclinic bifurcation. Additionally, the Jacobian matrix around the equilibrium point given by Eq. (7) presents a zero eigenvalue of multiplicity two.

\section{DICKE MODEL-LC CIRCUIT ANALOGY}

In recent years, electronic analogies have been successfully used to study key concepts and/or explore new focuses of complex quantum phenomena. In particular, they allowed for building experimental systems that offer certain implementation advantages - such as cost, complexity, and measuring protocols-over the original setups [51-54]. It is because of these features that electronic circuits have arisen as a powerful tool for exploring observables that obey the same mathematical description as those of the original quantum or classical systems. It is worth mentioning that while there are novel realizations of the quantum Dicke model employing superconducting qubits and oscillator circuits [55-57] to the best of our knowledge this is the first time the classical Dicke model is physically implemented.

Interestingly, the Hamiltonian (2) can be understood as two harmonic oscillators, one of them representing the set of two-level atoms $(P, Q)$ and the other the radiation field $(p, q)$, nonlinearly coupled through its atomic dipolar moment. This allows us to map the original Hamiltonian (2) to an electrical version of the harmonic oscillator, that is, LC circuits which are nonlinearly coupled to each other, with $\mathrm{L}$ standing for inductance, and $\mathrm{C}$ for capacitance. We can thus rewrite the Hamiltonian (2) in terms of the electrical variables by mapping $(p, q, P, Q) \rightarrow\left(\tilde{V}_{C_{1}}, \tilde{I}_{L_{1}}, \tilde{V}_{C_{2}}, \tilde{I}_{L_{2}}\right)$ with $\tilde{V}_{C_{1}}=\sqrt{\omega C_{1}} V_{C_{1}}$, $\tilde{I}_{L_{1}}=\sqrt{\omega L_{1}} I_{L_{1}}, \tilde{V}_{C_{2}}=\sqrt{\omega_{0} C_{2}} V_{C_{2}}$, and $\tilde{I}_{L_{2}}=\sqrt{\omega_{0} L_{2}} I_{L_{2}}$, where $\tilde{V}_{C_{j}}$ is the normalized voltage in the $j$ th capacitor, and $\tilde{I}_{L_{j}}$ is the normalized current passing through of the $j$ th inductor, with $j=1,2$. The system described by the electrical variables can then be experimentally implemented using active electrical networks of capacitors, resistors, operational amplifiers, and analog multipliers (see Appendix A for details). In our experimental setup, the parameters and the initial conditions are established by controllable voltage levels, in other words, the values of $\omega, \omega_{0}, \gamma, \tilde{V}_{C_{1}}(0), \tilde{V}_{C_{2}}(0), \tilde{I}_{L_{1}}(0)$, and $\tilde{I}_{L_{2}}(0)$ are directly mapped to electrical potential differences provided by high-resolution digital-to-analog converters.

To explore the dynamical evolution of the classical model under different conditions, we follow the voltages of each oscillator (voltage in capacitors and current through inductors) corresponding to the canonical variables $(p, q, P, Q)$ to collect information about the energy of the system. We extract time series of $300 \mathrm{~s}$ sampled at $15 \mathrm{~ms}$ using an oscilloscope Tektronix TBS200 (impedance $1 \mathrm{M} \Omega$ ). Electronic components were mounted and soldered on a printed circuit board $(15 \times$ $15 \mathrm{~cm}$ ) to avoid faulty contacts and poor stability.

\section{RESULTS}

One of the merits of the proposed setup is the possibility of exploring many remarkable features of the classical Dicke model by easily accessible parameter configurations. To illustrate the functionality of our experimental setup, we carry out measurements of some representative phenomena of the Hamiltonian (2). In what follows, we compare experimental and theoretical results for the ground-state and excited-state phase transition, and the dynamics of different regular and chaos regions, all in the resonant case $\left(\omega=\omega_{0}\right)$. Additionally, we calculate the finite-time Lyapunov exponent from the measured temporal grow of the classical equivalent of the FOTOC.

\section{A. Ground-state phase transition}

In the thermodynamic limit, i.e., when the number of atoms $N$ goes to infinity, the Dicke model presents a quantum phase transition (whenever the interaction strength reaches a critical value) characterized by a discontinuity in the second derivative of the minimal energy [58]. This can be observed by following the ground-state energy of our experimental setup and comparing it to the semiclassical ground-state energy $E_{0}(\gamma)$ given by

$$
E_{0}(\gamma)=\left\{\begin{array}{cc}
-\omega_{0} & \text { for } \quad \gamma \leqslant \gamma_{c}, \\
-\frac{\omega_{0}}{2}\left(\frac{\gamma_{c}^{2}}{\gamma^{2}}+\frac{\gamma^{2}}{\gamma_{c}^{2}}\right) & \text { for } \quad \gamma>\gamma_{c} .
\end{array}\right.
$$

The averaged experimental energy along a given trajectory $\langle E\rangle$ was calculated using the measured data of our experimental setup through the following expression:

$$
\langle E\rangle=\frac{1}{t} \int_{0}^{t} H_{c l} d \tau,
$$

where $t$ corresponds to the observation time. Figure 1(a) shows the classical ground-state energy of the classical Dicke model, as a function of the interaction strength, for both experimental measurements (black points) and the analytical expression in Eq. (8) (red line). Qualitative and quantitative agreements between both results can clearly be observed.

We can further use the measured data to plot the classical analog of the mean photon number $\langle n\rangle$ and the atomic inversion $\left\langle j_{z}\right\rangle$ as a function of the interaction strength. They are given by $\left(p^{2}+q^{2}\right) / 2$ and $\left(P^{2}+Q^{2}\right) / 2$, respectively [see Figs. 1(b) and 1(c)]. Note that the ground state undergoes a sudden change in its properties, going from an unexcited normal phase $\left(\gamma \leqslant \gamma_{c}\right)$ with no photons and no excited atoms to a symmetry-broken superradiant phase $\left(\gamma>\gamma_{c}\right)$, in which both the mean number of photons and the number of excited atoms become comparable to the total number of atoms in the system. In other words, the field and atomic excitations acquire macroscopic occupations. This transition is an example of a quantum collective behavior and has a close connection with entanglement and quantum chaos [33]. 

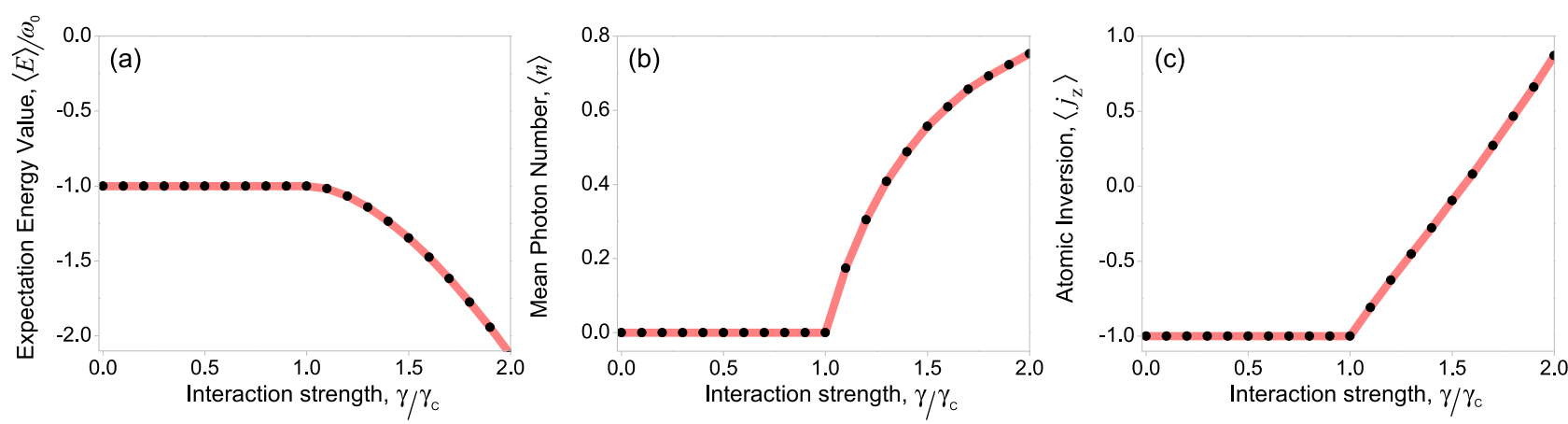

FIG. 1. (a) Classical analog of the ground-state energy. (b) Classical analog of the mean photon number $\langle n\rangle$. (c) Classical analog of the atomic inversion $\left\langle j_{z}\right\rangle$, as a function of the interaction strength, for the classical Dicke model. The black points correspond to experimental data, whereas the red lines denote theoretical results. The parameters are set to $\omega=1$ and $\omega_{0}=1 \mathrm{~s}^{-1}$. The standard deviation of the experimental results are inside the black points.

\section{B. Regularity and chaos}

In this subsection, we explore the presence of regularity and chaos for different energies. By employing the Lyapunov exponents and phase space, we determine the nature of the trajectories for both theoretical and experimental results. Figure 2(i) shows the map of the average Lyapunov exponents [59-61] as a function of the energy $E$ and the coupling strength $\gamma / \gamma_{c}$. For each pair $\left(E, \gamma / \gamma_{c}\right)$ the average Lyapunov exponent $\bar{\lambda}$ is calculated by simultaneously solving the dynamical equations (5) and those in the corresponding tangent space for 1000 of the initial conditions in the restricted phase space defined by $E$ and $p=0$ [42]. The thick black line denotes the ground-state energy and the red dot the critical point $\gamma_{c}=\sqrt{\omega \omega_{0}} / 2$, which marks the transition between the normal and superradiant phase. Note that the range of possible energies $E$ is lower bounded by the ground state [gray zone in Fig. 2(i)]. There is no upper bound in the energy because the number of bosons in the field is not limited.

By looking at the map of the average Lyapunov exponents, one can appreciate the rich phenomenology in terms of regularity and chaos. We selected four different energies, in resonance and for the interaction strength $\gamma=2 \gamma_{c}$. Each value is indicated on the phase map by white points: (a) $E=-0.5$, (b) $E=-1.1$, (c) $E=-1.5$, and (d) $E=-1.8$.

Figure 2(ii) displays a detailed map of chaoticity in the plane $(Q, P)$, for $p=0$ and $E=-1.5$, a mixed zone where regular and chaotic orbits coexist. Four different initial conditions $(p, q, P, Q)$ are selected and marked with white dots, as representatives of the different dynamics.

The projections of the trajectories in the planes $(q, p)$ and $(Q, P)$ for the numerical simulations, and in the planes $\left(\tilde{I}_{L_{1}}, \tilde{V}_{C_{1}}\right)$ and $\left(\tilde{I}_{L_{2}}, \tilde{V}_{C_{2}}\right)$ for the experimental results, corresponding to the initial points Figs. 2(a)-2(d), are shown on the right-hand side of Fig. 2. For energies larger than $E=-1$, the dynamics are fully chaotic in an ergodic sense. At these energies, strong chaos characterized by Lyapunov exponents beyond $\lambda=0.15$ can be found. Note that $\lambda$ refers to the Lyapunov exponent for each initial condition shown in Fig. 2(ii); whereas $\bar{\lambda}$ in Fig. 2(i) refers to the average of all initial conditions. Figure 2(a) shows a dense phase plane for $E=-0.5$, indicating the presence of chaotic dynamic. For energies close to $E=-1$, chaotic trajectories predominate.
As Figure 2(b) shows, the phase plane also exhibits a chaotic trajectory for $E=-1.1$. When the energy decreases up to $E=-1.5$, the complexity and nonlinearity of the system acquire relevance in the dynamics, thus giving rise to a mixed zone where chaos and regularity coexist. Figures 2(c.1) to 2(c.4) display phase planes for the subcases marked into the map of chaoticity [Fig. 2(ii)]. Note that Figs. 2(c.1) and 2(c.3) show regular dynamics; whereas the planes in Figs. 2(c.2) and 2(c.4) present chaotic behavior. Finally, when $E=-1.8$, a limit cycle is formed, shown in Fig. 2(d). To quantify the nature of the experimental trajectories, we computed the largest Lyapunov exponents $\left(\lambda_{\mathrm{EXP}}\right)$ by applying the method described in [62] and Appendix B. For comparison purposes, we also calculated theoretical Lyapunov exponents $\left(\lambda_{\mathrm{THE}}\right)$. The results are summarized in Table I. From our previous studies [42], the values $\lambda<0.004$ are considered null because they are below the numerical error.

Interestingly, in recent years, it has been shown that there exists a strong connection between the classical Lyapunov exponent and a useful measure of quantum chaos known as the out-of-time-order correlator or OTOC [31,44]. In quantum systems, the OTOC quantifies the degree of noncommutativity between two operators $\hat{W(t)}$ and $V \hat{(0)}$. Selecting $\hat{W(t)}=$ $e^{i \delta \phi G(t)}$, with $\hat{G}$ an Hermitian operator, and $\hat{V}$ as the projection operator onto an initial state $\left|\Psi_{0}\right\rangle$, a special type of fidelity OTOC (FOTOC) can be defined [31]. If $\delta \phi \ll 1$, this FOTOC concurs with the variance of $\hat{G}$ [31]. As the exponential grow of the FOTOC takes place in times shorter than the Ehrenfest time, its dynamics can be computed with semiclassical phase

TABLE I. Lyapunov exponents.

\begin{tabular}{lccc}
\hline \hline & $E$ & $\lambda_{\text {EXP }}$ & $\lambda_{\text {THE }}$ \\
\hline (a) & -0.5 & 0.289 & 0.295 \\
(b) & -1.1 & 0.118 & 0.124 \\
(c. 1$)$ & -1.5 & 0.000 & 0.000 \\
(c.2) & -1.5 & 0.009 & 0.011 \\
(c.3) & -1.5 & 0.001 & 0.001 \\
(c.4) & -1.5 & 0.011 & 0.012 \\
(d) & -1.8 & 0.000 & 0.000 \\
\hline \hline
\end{tabular}




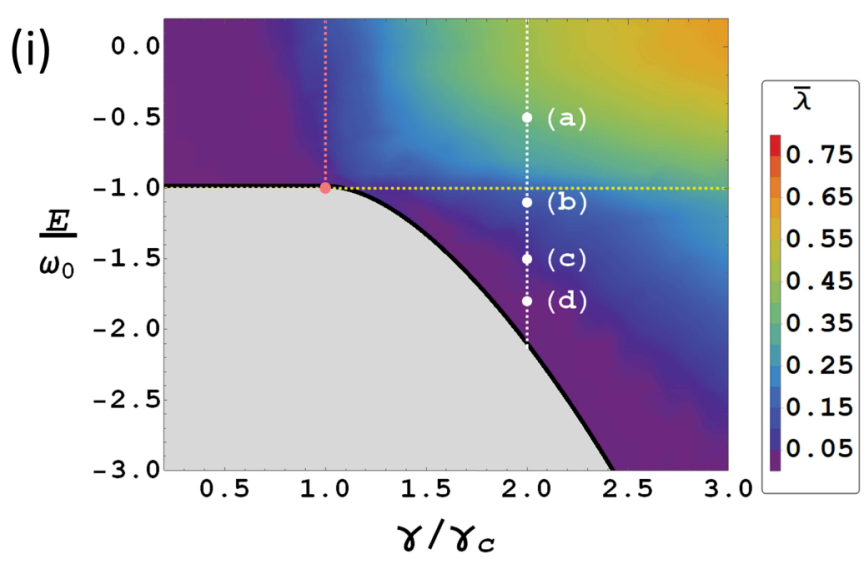

(a)

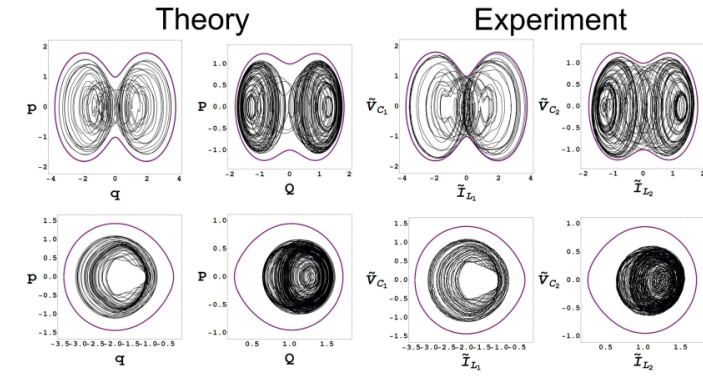

(ii)
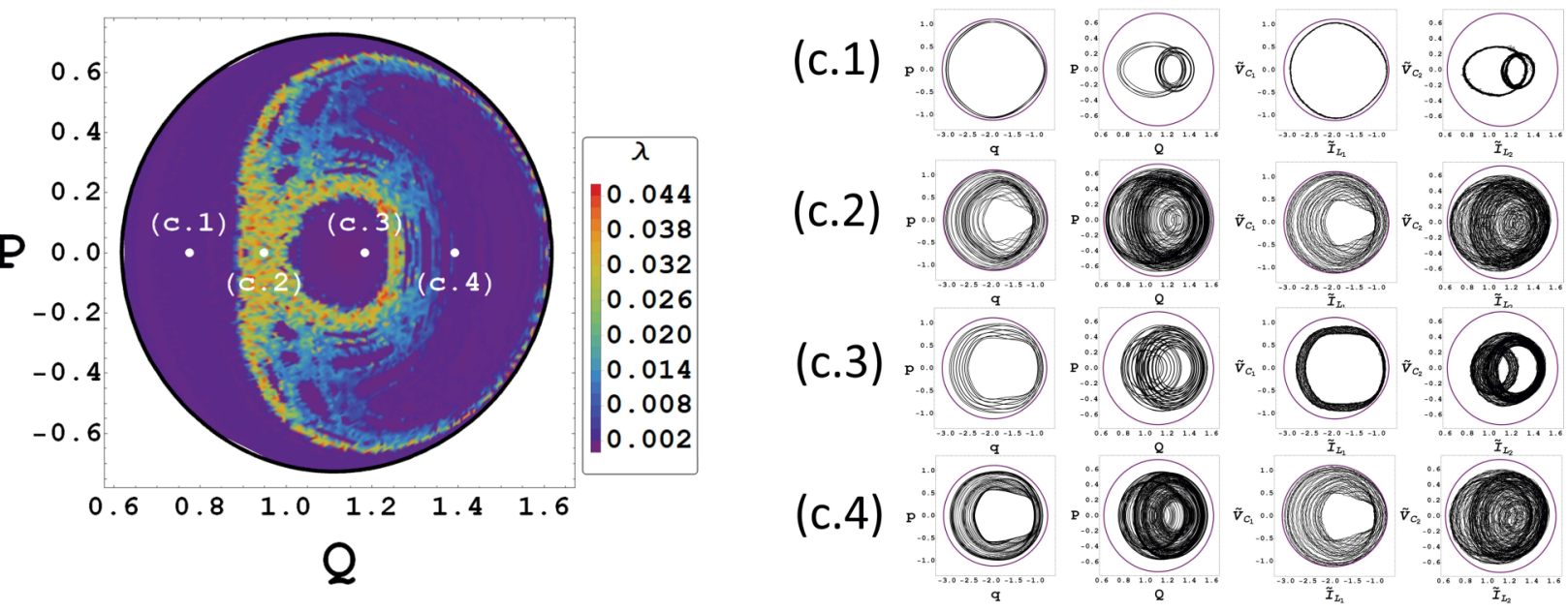

FIG. 2. (i) Map of the average Lyapunov exponents as a function of the energy $E$ and the coupling strength $\gamma / \gamma_{c}$. The thick black line follows the ground-state energy and the red dot denotes the critical point $\gamma_{c}$. The white dots indicate four different energies for a fixed coupling $\gamma=2 \gamma_{c}$ : (a) $E=-0.5$, (b) $E=-1.1$, (c) $E=-1.5$, and (d) $E=-1.8$. (ii) Map of chaoticity in the plane ( $Q$, $P$ ), for $p=0$ and $E=-1.5$ (mixed chaos zone). Four different initial conditions are marked with white points into the map of chaoticity. Right-hand side shows the projections of the trajectories in the planes $(q, p)$ and $(Q, P)$ for the numerical simulations, and in the planes $\left(\tilde{I}_{L_{1}}, \tilde{V}_{C_{1}}\right)$ and $\left(\tilde{I}_{L_{2}}, \tilde{V}_{C_{2}}\right)$ for the experimental results, corresponding to the initial points $(p, q, P, Q)$ : (a) $(0,-0.13372,0,1.22474)$, (b) $(0,-0.50471,0,1.22474), \quad(c .1)(0,-0.76496,0,0.7746), \quad(c .2)(0,-0.72693,0,0.94868)$, (c.3) $(0,-0.79433,0,1.18322),($ c.4) $(0,-1,0,1.41421)$, and (d) $(0,-1.0996,0,1)$. The purple lines in the phase planes denote the available evolution space. The time evolution runs up to $300 \mathrm{~s}$, and we set $\omega=1 \mathrm{~s}^{-1}$ and $\omega_{0}=1 \mathrm{~s}^{-1}$.

space methods, such as the truncated Wigner approximation (TWA) [63-65]. The TWA allows to compute the short-time quantum dynamics using the classical equations of motion, averaging the observable over a set of initial conditions. The random sampling reproduces the quantum fluctuations of a quantum initial state, usually taken from a Gaussian distribution, which for the Dicke model have a width of $2 / N$ for each degree of freedom, where $N$ is the number of atoms [31].

Because the dynamics of both quantum and classical variances are the same until saturation [66] within the context of the TWA, in this subsection, we compute the temporal evolution of the variance of the measured voltages, a classical analog of the FOTOC, whose fast grow at short times has an exponent, known as finite-time Lyapunov exponent (FTLE) and denoted as $\Lambda_{C}$. It is calculated for an ensemble of 256 initial conditions in the superradiant phase (chaotic energy region) by employing experimental data of the electrical coordinate $\sigma^{2}\left(\tilde{I}_{L_{1}}\right)$.
To allow for a direct comparison between the classical FTLE of our experimental realization with the planned measurements of the FOTOC in the ongoing experiments with ion traps, seeking to investigate quantum chaos in the Dicke model, we employ the same parameters used in [30,31]. Figure 3(a) shows the temporal evolution of the normalized current in one of the inductors $\left(\tilde{I}_{L_{1}}\right)$, considering 256 randomly distributed initial conditions, which were acquired from our experimental setup. The red solid line represents $\left\langle\tilde{I}_{L_{1}}\right\rangle$ along the time. Random initial conditions are generated by the electronic noise, which creates fluctuations in the voltages, making the system explore a region in phase space whose size is proportional to these fluctuations.

Figure 3(b) shows the averaged evolution of the variance of the electrical coordinate $\sigma^{2}\left(\tilde{I}_{L_{1}}\right)$ as a function of time. The FTLE growth rate $\Lambda_{C}$ is obtained by fitting the curve with a monomial function indicated by the black solid line in the same figure. Note that this is the same procedure as the one reported in [44]. The slope of the linear regression is 

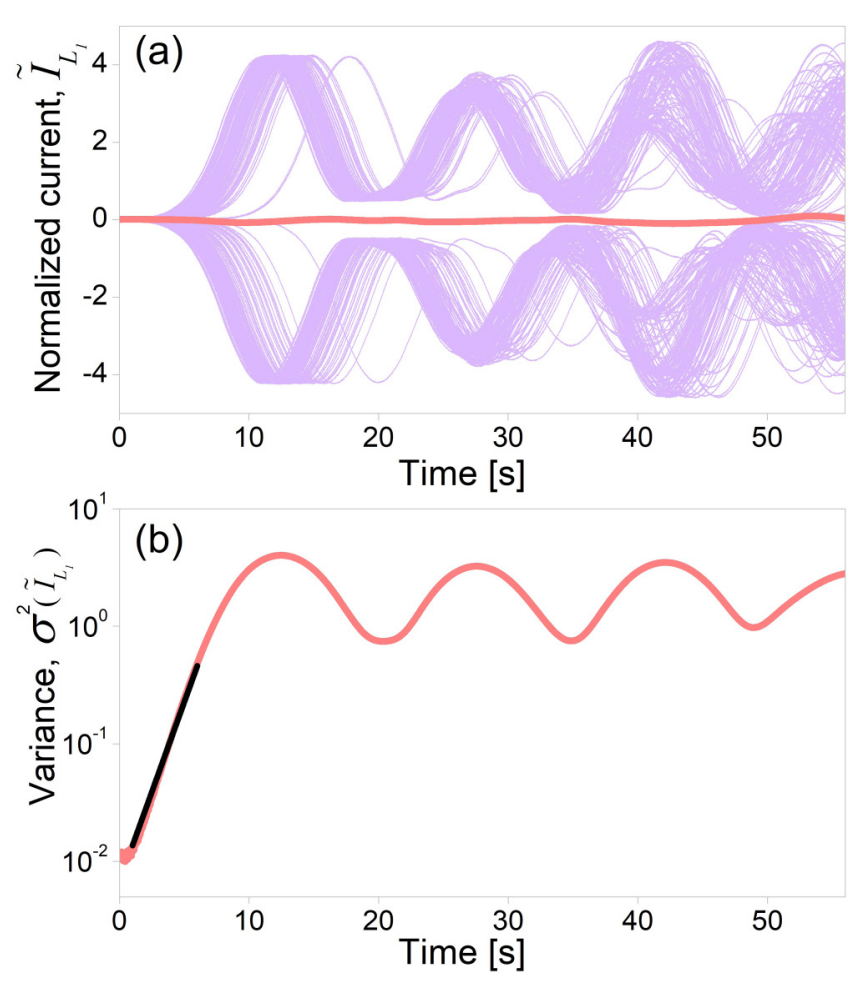

FIG. 3. (a) Experimentally measured time series for 256 randomly distributed initial conditions (purple lines) for the normalized current passing through one of the inductors $\left(\tilde{I}_{L_{1}}\right)$. Red line corresponds to the mean current as a function of time. (b) Variance of the normalized current $\sigma^{2}\left(\tilde{I}_{L_{1}}\right)$ as a function of time. Black line denotes the associated fit for $\sigma^{2}\left(\tilde{I}_{L_{1}}\right)$ in the short-time behavior. The time evolution runs up to $60 \mathrm{~s}$, and we set $\omega=0.5 \mathrm{~s}^{-1}, \omega_{0}=0.7 \mathrm{~s}^{-1}$, and $\gamma=0.66$.

$\Lambda_{C}=0.2865$. At long times, the FTLE saturates and fluctuates around its asymptotic value, due to the finite size of the phase plane. In this regime, the quantum-classical correspondence no longer holds. For comparison purposes, we also calculate the mean Lyapunov exponent by averaging over the resulting Lyapunov exponents of the 256 initial conditions. We obtained a mean Lyapunov exponent of $\hat{\lambda}=$ 0.1147 , which is nearly two times smaller than the grown rate of the FOTOC as was predicted in [31]. This confirms the quantitative correspondence between both indicators.

\section{Excited-state phase transition}

Similarly to the transition experienced by the ground state, the classical Dicke model exhibits an excited-state quantum phase transition (ESQPT), which occurs along the energy spectrum for fixed parameters of the Hamiltonian. This phenomenon denotes a singularity in the spectrum of quantized energy levels of the system which affects both density and dynamics levels [67], in fact, the ESQPT can be identified by studying the density of states in the semiclassical limit. Interestingly, such singularity leads to dramatic dynamical consequences [46].

Note that the gap between the ground state and critical energy $E=-1$ [dotted white horizontal line in Fig. 2(i)] vanishes as the interaction strength decreases. Interestingly, at $\gamma=\gamma_{c}$ and $E=-1$, two of three critical points become complex and $\mathrm{K}_{0}$ point is the only real one; in other words, the system goes from three equilibrium points in the superradiant phase to only one in the normal phase. Importantly, the critical points provide useful information to follow the behavior of the energy minimum, and their stability changes give rise to dramatic alterations of the accessible phase plane. Such alterations unveil the presence of an ESQPT. In the Dicke model, for energies in the subcritical case $(E<-1)$, the available phase plane is formed by two disconnected lobes. At the critical energy value $E=-1$, both lobes collide at the point $(0,0,0,0)$. Finally, for energies in the supercritical case $(E>-1)$, these lobes merge, allowing the trajectories freely evolve in both lobes.

In the Dicke model, ESQPT is identified by studying the stability conditions of $X_{\mathbb{O}}$ point as a function of the interaction strength. This point goes from a center point in the normal phase to a hyperbolic point in the superradiant phase. It is clear that the initial condition $(p, q, P, Q)=(0,0,0,0)$ leads to a stationary solution irrespective of $\gamma$ value due to the fact that it is located just at the equilibrium. When the interaction strength is smaller than its critical value, for any arbitrarily small perturbation $\delta \mathbb{X}=(\delta p, \delta q, \delta P, \delta Q)$ around $\mathbb{K}_{\mathbb{O}}$ (center point), the system presents stationary solutions. Beyond the critical interaction strength, $\mathbb{K}_{\mathbb{O}}$ becomes a hyperbolic point, which for any perturbed state $\delta \mathbb{X}$ escapes out of the equilibrium, exhibiting irregular oscillatory solutions.

Figure 4 shows the temporal evolution of the electrical coordinates $\left(\tilde{V}_{C_{1}}, \tilde{I}_{L_{1}}, \tilde{V}_{C_{2}}, \tilde{I}_{L_{2}}\right)$ measured for arbitrarily small perturbed initial conditions, considering three different interaction strengths: (a) $\gamma=0.9 \gamma_{c}$ (normal phase), (b) $\gamma=\gamma_{c}$ (QPT), and (c) $\gamma=1.1 \gamma_{c}$ (superradiant). As predicted, for couplings below $\gamma_{c}$, all solutions are stationary, that is, the trajectories converge to the equilibrium point $\mathbb{X}_{\mathbb{D}}$, as depicted in Fig. 4(a). For $\gamma=\gamma_{c}$, exactly at the QPT, the solutions are yet stationary but the time series begin to exhibit slight alterations as shown in Fig. 4(b). In striking contrast, when the couplings becomes larger than $\gamma_{c}$, any small perturbation leads to irregular trajectories, specifically chaotic dynamics [see Fig. 4(c)]. There, the initial condition does not converge to the equilibrium point, thus making evident the instability of the hyperbolic point. We would like to stress that the electronic noise associated with the electrical components in our experimental setup, introduces an unavoidable perturbation in the initial state (see Appendix A). In this way, it is not necessary to physically prepare a perturbed initial condition in the system, the stability or instability of the critical point is naturally unveiled by the electronic fluctuations of the device. In the normal phase, for energies at $E=-1$, as the cases shown in Figs. 1, 4(a) and 4(b), the electronic noise forces the trajectories to fluctuate around the equilibrium point $\mathbb{X}_{\mathbb{O}}$. The measured voltages are not fixed in $\mathfrak{X}_{\mathbb{O}}$, but randomly take values inside a four-dimensional sphere with radius equal to the standard deviation of the noise, which is found to be $\sim 15 \mathrm{mV}$.

\section{v. CONCLUSION}

In summary, we implemented the electrical version of the classical Dicke model by means of active, synthetic electrical 

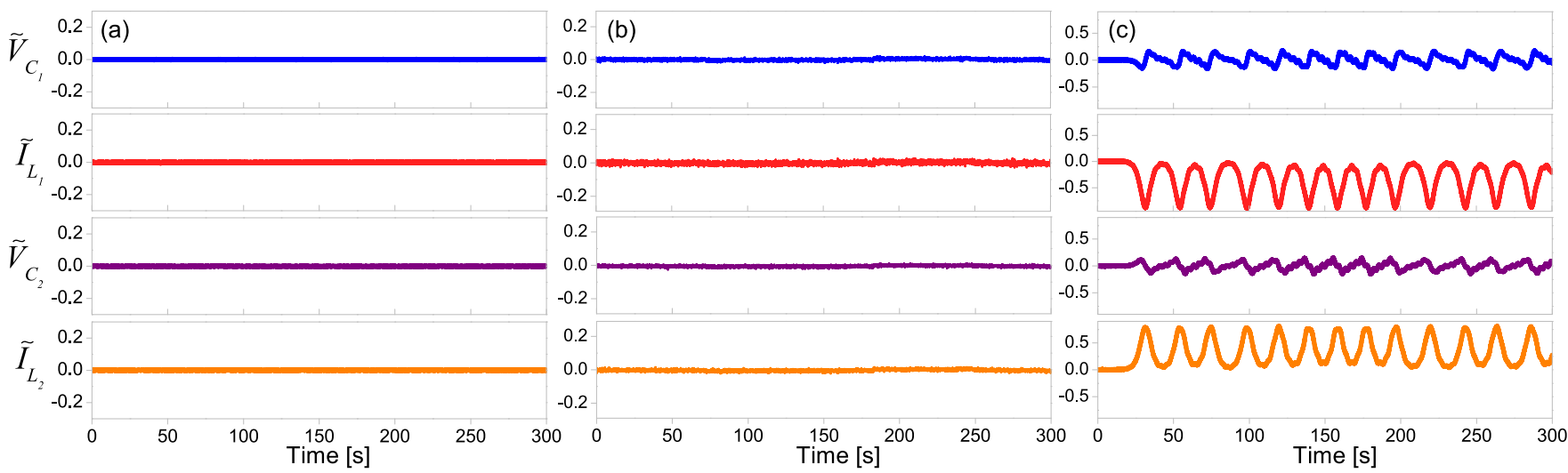

FIG. 4. Temporal evolutions of the electrical coordinates $\left(\tilde{V}_{C_{1}}, \tilde{I}_{L_{1}}, \tilde{V}_{C_{2}}, \tilde{L}_{L_{2}}\right)$, obtained from experimental data for three cases: (a) Normal phase $\left(\gamma=0.9 \gamma_{c}\right)$ with $E_{0}=-0.99997 \omega_{0}$, (b) QPT $\left(\gamma=\gamma_{c}\right)$ with $E_{0}=-0.999984 \omega_{0}$, and (c) superradiant phase $\left(\gamma=1.1 \gamma_{c}\right)$ with $E_{0}=$ $-0.999865 \omega_{0}$.

networks. Our setup makes use of two nonlinearly coupled active LC oscillators, where the system parameters $\omega, \omega_{0}$, $\gamma$, and initial conditions are independently controlled by voltage signals, thus making the configurations of the system easily accessible, and capable of exploring many remarkable features of the Dicke model. We demonstrated that our experimental setup allows for the direct observation of both phase transitions as well as the exploration of a wide range of excitation energies and Hamiltonian parameters, unveiling the different dynamical regimes, from regular to fully chaotic. Experimental phase planes and Lyapunov exponents were used to study regular and chaotic trajectories. The high resolution of our measuring equipment allowed to show the existence of a phase transition by following the classical analogs of the mean photon number and the atomic inversion as a function of the coupling parameter. In particular, we showed that the ground state goes from an unexcited normal phase (no photons and no excited atoms) to a symmetry-broken superradiant phase (field and atomic excitations acquire macroscopic occupations). Also, we revealed the presence of an excited-state phase transition by studying the stability conditions of the equilibrium point $\mathbb{X}_{0}$ for small perturbed initial conditions, as a function of the interaction strength. $\mathbb{K}_{0}$ point goes from a center point $\left(\gamma<\gamma_{c}\right)$ to a hyperbolic point $\left(\gamma \geqslant \gamma_{c}\right)$, yielding dramatic alterations in the available phase plane. Interestingly, in our experimental setup, the initial perturbations are produced by the intrinsic electronic noise in the device, which allows us to define a "classical uncertainty region." In all cases, exhaustive numerical simulations were performed to show quantitative and qualitative agreement between theory and experiment. Finally, motivated by the current interest in the FOTOC growth rate as a quantum signature of chaos, we calculated for an ensemble of experimental trajectories the temporal grow of the variance of one measured voltage, as a classical analog of the FOTOC. Remarkably, the resulting growth rate coincides with the classical Lyapunov exponent, thus demonstrating that, indeed, our nonlinear electrical oscillators display all important features of the classical Dicke model. Because of its simplicity, our experimental setup constitutes a remarkable platform for exploring the richness of non-linear systems in terms of chaos and regularity.

\section{ACKNOWLEDGMENTS}

This work was supported by CONACyT-México under the project CB-2016-01/284372 and by DGAPA-UNAM under the project UNAM-PAPIIT IN102920. J.C.-C. and J.G.H. thank partial financial support from DGAPA-UNAM Grants No. IN109417 and IN104020. M.A.Q.-J. and J.L.A. thankfully acknowledge financial support by CONACyT-México under the project A1-S-8317.

\section{APPENDIX A: ELECTRONIC DESIGN OF THE CLASSICAL DICKE MODEL}

In this Appendix we provide a detailed description of the electronic implementation of the classical Dicke model. We start by writing the classical Hamiltonian (2) in terms of electrical variables, considering the map: $(p, q, P, Q) \rightarrow$ $\left(\tilde{V}_{C_{1}}, \tilde{I}_{L_{1}}, \tilde{V}_{C_{2}}, \tilde{I}_{L_{2}}\right)$. Thus, Eq. (2) becomes

$$
\begin{aligned}
H= & \frac{\omega}{2}\left(\tilde{V}_{C_{1}}^{2}+\tilde{I}_{L_{1}}^{2}\right)+\frac{\omega_{0}}{2}\left(\tilde{V}_{C_{2}}^{2}+\tilde{I}_{L_{2}}^{2}\right) \\
& +\gamma \tilde{I}_{L_{1}} \tilde{I}_{L_{2}} \sqrt{4-\tilde{V}_{C_{2}}^{2}-\tilde{I}_{L_{2}}^{2}}-\omega_{0},
\end{aligned}
$$

where $\omega^{2}=1 / L_{1} C_{1}$ and $\omega_{0}^{2}=1 / L_{2} C_{2}$ define the natural frequencies for the first and second oscillators, respectively. If we set $\left|L_{1}\right|=\left|C_{1}\right|$ and $\left|L_{2}\right|=\left|C_{2}\right|$, then $\omega=1 /\left|L_{1}\right|=1 /\left|C_{1}\right| \mathrm{s}^{-1}$ and $\omega_{0}=1 /\left|L_{2}\right|=1 /\left|C_{2}\right| \mathrm{s}^{-1}$. To explore the dynamical properties of the Hamiltonian (A1), we obtain the associated classical equations of motion, which read

$$
\begin{aligned}
C_{1} \dot{\tilde{V}}_{C_{1}} & =-\gamma C_{1} \tilde{I}_{L_{2}} \sqrt{4-\tilde{V}_{C_{2}}^{2}-\tilde{I}_{L_{2}}^{2}}-\tilde{I}_{L_{1}}, \\
L_{1} \dot{\tilde{I}}_{L_{1}} & =\tilde{V}_{C_{1}}, \\
C_{2} \dot{\tilde{V}}_{C_{2}} & =\frac{\gamma C_{2} \tilde{I}_{L_{1}} \tilde{I}_{L_{2}}^{2}}{\sqrt{4-\tilde{V}_{C_{2}}^{2}-\tilde{I}_{L_{2}}^{2}}}-\gamma C_{2} \tilde{I}_{L_{1}} \sqrt{4-\tilde{V}_{C_{2}}^{2}-\tilde{I}_{L_{2}}^{2}}-\tilde{I}_{L_{2}}, \\
L_{2} \dot{\tilde{I}}_{L_{2}} & =\tilde{V}_{C_{2}}-\frac{\gamma L_{2} \tilde{V}_{C_{2}} \tilde{L}_{L_{1}} \tilde{L}_{L_{2}}}{\sqrt{4-\tilde{V}_{C_{2}}^{2}-\tilde{I}_{L_{2}}^{2}}} .
\end{aligned}
$$

Our setup reproduces the dynamics of the motion equations described by Eq. (A2) (which effectively corresponds to a 

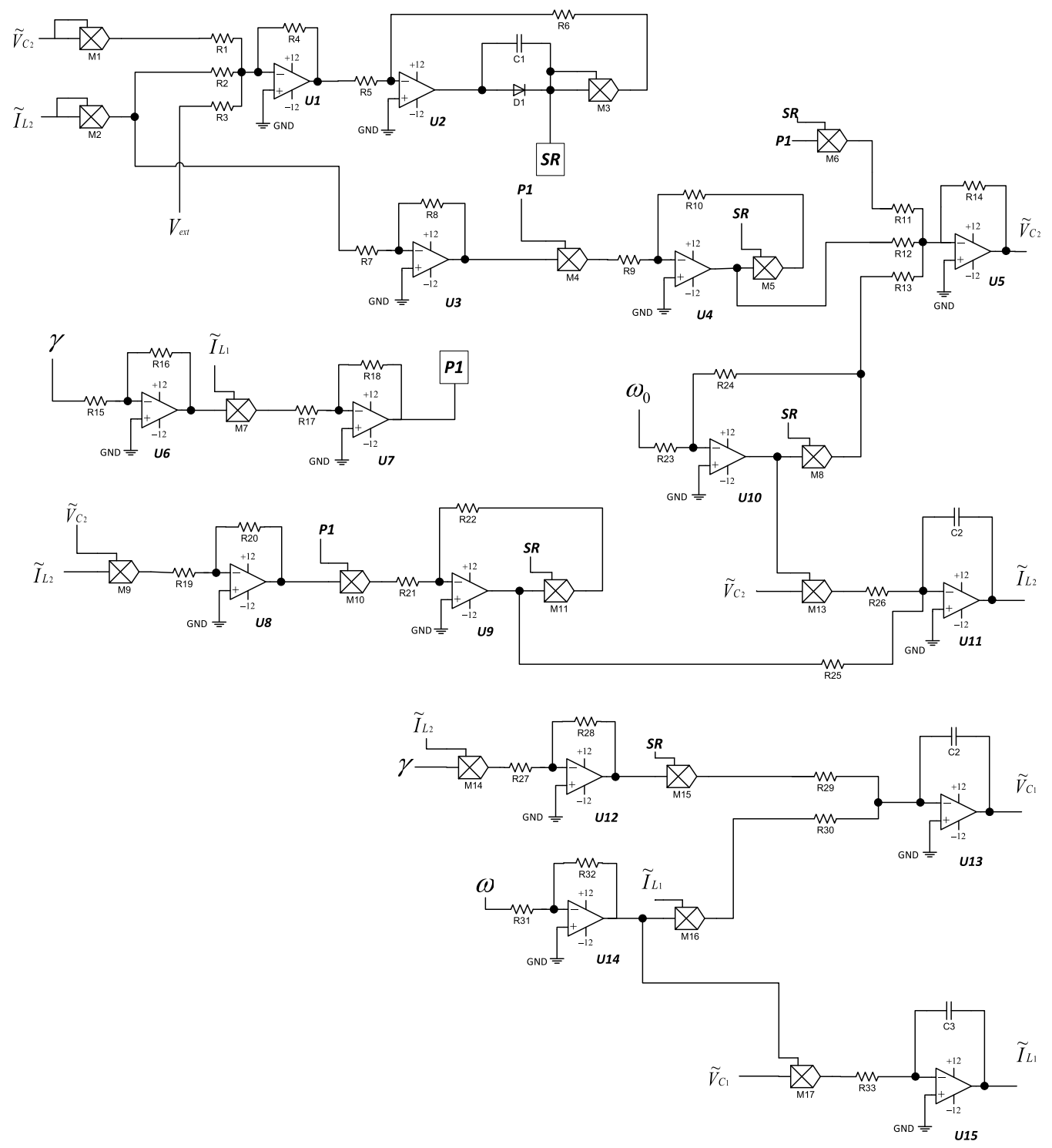

FIG. 5. Scheme of the electronic circuit of two nonlinearly coupled LC oscillators, described by Eq. (A2). The values for the resistors and capacitors are given in the text. The labels $S R$ and $P 1$ refer to interconnection of internal signals; whereas GND refers to the ground.

pair of nonlinearly coupled LC oscillators) using active electrical networks of operational amplifiers (OPAMPs). These networks consist of interconnecting resistors, capacitors, and OPAMPs to conform basic electrical networks where their voltage transfer functions are analogous to the mathematical operations. Thus, with few passive components around the operational amplifiers, it is possible to perform operations of addition, subtraction, integration, and amplification. Although these types of networks are commonly used to perform linear operations, a large number of active components can also implement nonlinear operations, such as logarithms, antilogarithms, and products between variables. A drawback of nonlinear configurations is their high temperature dependence which can lead to functional problems [68]. A possible solution to implement nonlinear functions consists in employing integrated analog multipliers. In fact, analog multipliers AD633JN (four-quadrant voltage multipliers chips with a typical error less than $1 \%$ ) were used to implement the nonlinear terms of our model, such as squaring, square rooting, multiplication, and division between variables.

The electronic implementation of Eq. (A2) presents two difficulties: (1) some variables exhibit amplitudes that exceed power supply limits and (2) the system parameters $\left(\omega, \omega_{0}, \gamma\right)$, and initial conditions $\left(\tilde{V}_{C_{1}}, \tilde{V}_{C_{2}}, \tilde{I}_{L_{1}}, \tilde{I}_{L_{2}}\right)$ should be controlled in an accessible way. To solve the first problem, we adopt scaling factors in gain amplifier configurations to obtain a half-scale condition. Particularly, the analog multipliers normalize the input signals by a factor of about 10 . For the second problem, we introduce system parameters, and initial conditions, via voltage signals provided by digital-to-analog converters (MCP4921, resolution: 12 bits) which are communicated to a master 8-bits microcontroller (PIC18 family) by serial peripheral interface (SPI) protocol. Each voltage value can be individually configured via software. With this configuration we avoid variations in the properties of the electronic components of the device. The scheme of the electronic circuit is shown in Fig. 5. There, $R_{j}, C_{j}, U_{j}$, 


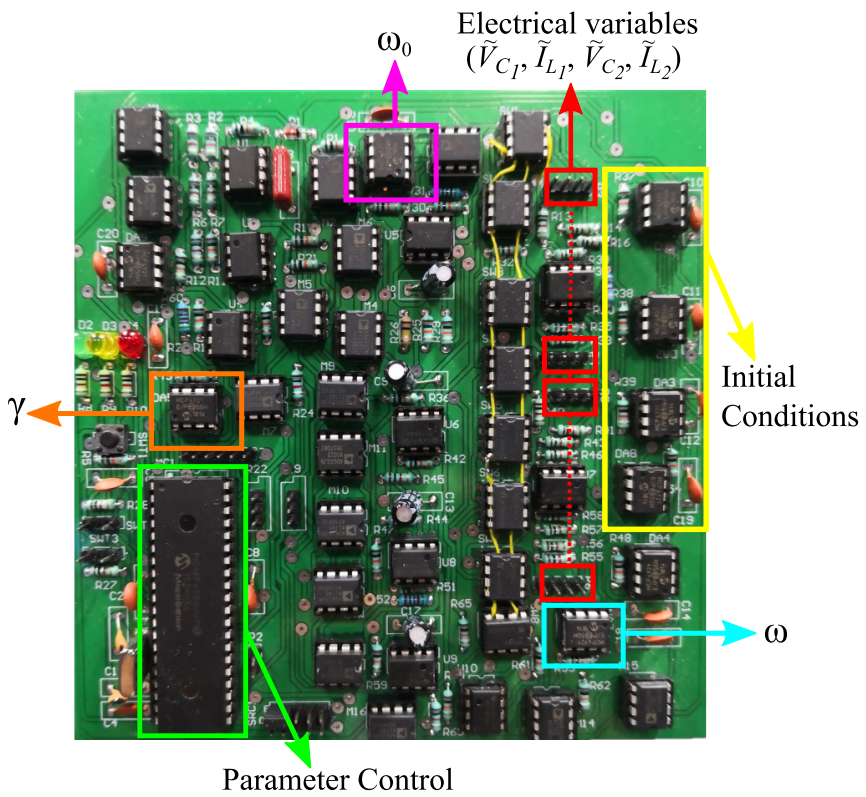

FIG. 6. Printed circuit board of the classical Dicke model.

and $M_{j}$ stand for resistors and capacitors, general-purpose operational amplifiers MC1458, and analog multiplier AD633JN, respectively. $D_{1}$ denotes a fast recovery diode $1 \mathrm{~N} 4148$. The resistor and capacitor values used in the circuit are the following: $R_{1}=R_{2}=R_{3}=R_{4}=R_{7}=R_{17}=R_{19}=$ $R_{27}=1 K \Omega, \quad R_{5}=R_{6}=R_{8}=R_{9}=R_{10}=R_{11}=R_{13}=$ $R_{16}=R_{18}=R_{20}=R_{21}=R_{22}=R_{24}=R_{25}=R_{26}=R_{28}=$ $R_{29}=R_{30}=R_{32}=R_{33}=10 \mathrm{~K} \Omega, \quad R_{12}=100 \mathrm{~K} \Omega, \quad R_{15}=$ $R_{23}=R_{31}=5 K \Omega C_{1}=0.01 \mu \mathrm{F}$, and $C_{2}=C_{3}=C_{4}=C_{5}=$ $10 \mu \mathrm{F}$. The labels $S R$ and $P 1$ refer to interconnection of internal signals.

Electronic components were mounted and soldered on a printed circuit board (PCB, $15 \times 15 \mathrm{~cm}$ ) to avoid faulty contacts and poor stability. Figure 6 shows the electronic circuit realization of the system (A2) on the PCB. In the same figure, the modules that control the parameters of the system are signaled with orange, cyan, and magenta squares, and the initial condition modules with yellow; all of them governed by a microcontroller (green square) via software. The red square indicates the output electrical variables $\left(\tilde{V}_{C_{1}}, \tilde{I}_{L_{1}}, \tilde{V}_{C_{2}}, \tilde{I}_{L_{2}}\right)$.

In spite of the robustness, flexibility, and good agreement with theoretical results, the proposed circuit is not ideal, but has a subtle interplay of gain and loss which becomes evident in the superradiant phase. The active electrical network presents a highly complex energy exchange, mainly due to the action of the operational amplifiers, which are all the time returning the system to its equilibrium point, recovering the dissipated energy by the circuit at short times, and thus demanding the required power from the supply source which polarizes them.

To show the energy behavior of the experimental setup, in Fig. 7, we plot the energy evolution as a function of time (orange lines) for three representative energies of Fig. 2: (b) $E=-1.1$ (chaotic region), (c.1) $E=-1.5$ (mixed zone), and (d) $E=-1.8$ (regular region). We also calculated the temporal averaged energy for each case (red lines) using the

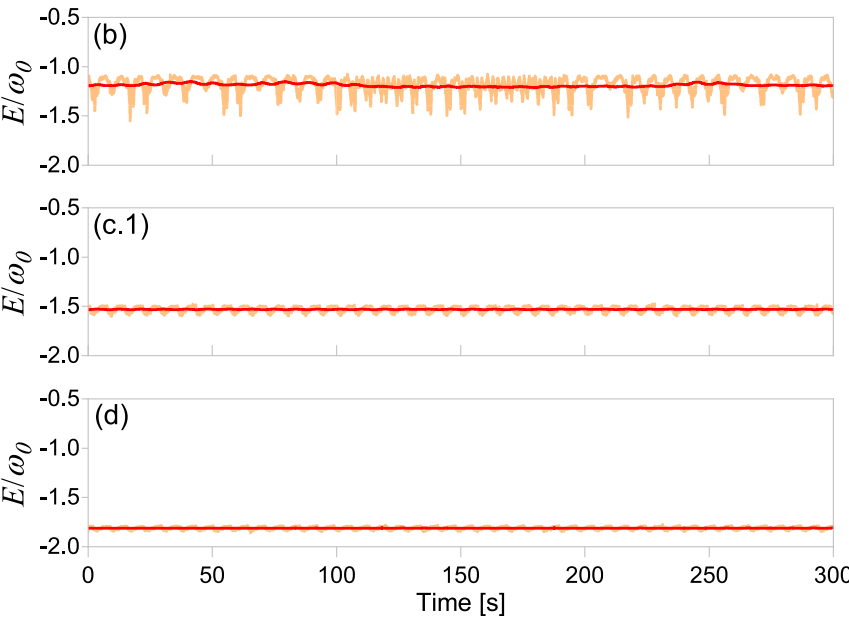

FIG. 7. Energy time series (orange) and temporal average (red) for small time windows of selected points given in Fig. 2, with initial energies (b) $E=-1.1$, (c.1) $E=-1.5$, and (d) $E=-1.8$.

moving average filtering method [69] for a time window of 10 seconds. Note that, in all cases, the energy fluctuates (orange lines) as a result of the continuous competence between dissipation and energy recovery in the circuit. However, the net effect of these fluctuations is that the mean energy is constant in time, but its value is slightly smaller than the initial energy. When $E=-1.1$ [see Fig. 7(b)], the mean energy deviates about $8 \%$ with respect to the initial value, whereas for the energies $E=-1.5$ and $E=-1.8$ shown in Figs. 7(c.1) and $7(d)$, respectively, such deviations are less than $3 \%$, making the effect hard to notice. Here, it is worth pointing out that our capacity to control parameters and initial conditions is not seen limited by the dissipation-gain effect.

\section{APPENDIX B: LYAPUNOV EXPONENT CALCULATION}

A notable property of a chaotic system is its high sensitivity to initial conditions, a fact that leads to the concept of the Lyapunov exponent, which has been used as an indicator of chaos for a long time. It measures the average exponential divergence of conditions infinitesimally nearby to the initial state. In general terms, there are as many Lyapunov exponents as the dimension of the phase plane. However, in many applications, it is sufficient to calculate only the largest Lyapunov exponent. A system with a positive largest Lyapunov exponent is defined to be chaotic [59-61].

In the subsection "Regularity and chaos," we estimated the largest Lyapunov exponent of each point of Figs. 2(a)2(d), using the experimental time series, that is, the temporal evolution of the variables $\left(\tilde{V}_{C_{1}}, \tilde{I}_{L_{1}}, \tilde{V}_{C_{2}}, \tilde{I}_{L_{2}}\right)$, following the proposed method by the authors of [62], which is fast and easy to implement. Below we summarize the algorithm.

Step 1. Reconstruction of the dynamics from a single time series. In our case, all state variables of the system are available, namely, $\left(\tilde{V}_{C_{1}}, \tilde{I}_{L_{1}}, \tilde{V}_{C_{2}}, \tilde{I}_{L_{2}}\right)$. The trajectory $\mathcal{X}$ of the system can be expressed as

$$
\mathbb{X}=\left\{x_{1}, x_{2}, \ldots, x_{M}\right\},
$$


where $x_{i}=\left[\tilde{V}_{C_{1}}\left(t_{i}\right), \tilde{I}_{L_{1}}\left(t_{i}\right), \tilde{V}_{C_{2}}\left(t_{i}\right), \tilde{I}_{L_{2}}\left(t_{i}\right)\right]$ is the state of the system at a discrete time $t_{i}$. M is the number of data points in the time series.

Step 2. Calculate the nearest neighbor of each point on the trajectory. The nearest neighbor $x_{\hat{i}}$ minimizes the Euclidean distance to the reference point $x_{i}$, that is,

$$
d_{i}=\min \left\|x_{i}-x_{\hat{i}}\right\| \text {. }
$$

There, the nearest neighbors should have a temporal separation greater than the mean period $\bar{T}$ of the time series $\left\|x_{i}-x_{\hat{i}}\right\|>\bar{T}$.

Step 3. The largest Lyapunov exponent is calculated using a least-squares fit to the average separation of neighbors defined by

$$
y\left(t_{i}\right) \approx \frac{1}{t_{s}}\left\langle\ln d_{j}\left(t_{i}\right)\right\rangle,
$$

where $t_{s}$ is the sampling period and $\langle$.$\rangle denotes the average$ over all values of $j$.

Exhaustive numerical simulations were performed by varying the number of data points of experimental time series, which were smoothed using the moving average filtering method [69]. From our results, we find that Lyapunov exponents with values close to theoretical values were obtained with a average time-window size equal to $750 \mathrm{~ms}$, and a time series of $120 \mathrm{~s}$.
[1] P. Reimann, Foundation of Statistical Mechanics under Experimentally Realistic Conditions, Phys. Rev. Lett. 101, 190403 (2008).

[2] A. J. Short, Equilibration of quantum systems and subsystems, New J. Phys. 13, 053009 (2011).

[3] A. J. Short and T. C. Farrelly, Quantum equilibration in finite time, New J. Phys. 14, 013063 (2012).

[4] P. R. Zangara, A. D. Dente, E. J. Torres-Herrera, H. M. Pastawski, A. Iucci, and L. F. Santos, Time fluctuations in isolated quantum systems of interacting particles, Phys. Rev. E 88, 032913 (2013).

[5] K. He, L. F. Santos, T. M. Wright, and M. Rigol, Single-particle and many-body analyses of a quasiperiodic integrable system after a quench, Phys. Rev. A 87, 063637 (2013).

[6] C. Gogolin and J. Eisert, Equilibration, thermalisation, and the emergence of statistical mechanics in closed quantum systems, Rep. Prog. Phys. 79, 056001 (2016).

[7] F. Borgonovi, F. M. Izrailev, L. F. Santos, and V. G. Zelevinsky, Quantum chaos and thermalization in isolated systems of interacting particles, Phys. Rep. 626, 1 (2016).

[8] L. D’Alessio, Y. Kafri, A. Polkovnikov, and M. Rigol, From quantum chaos and eigenstate thermalization to statistical mechanics and thermodynamics, Adv. Phys. 65, 239 (2016).

[9] P. Reimann, Typical fast thermalization processes in closed many-body systems, Nat. Commun. 7, 10821 (2016).

[10] P. Reimann, Dynamical Typicality Approach to Eigenstate Thermalization, Phys. Rev. Lett. 120, 230601 (2018).

[11] A. Dymarsky, N. Lashkari, and H. Liu, Subsystem eigenstate thermalization hypothesis, Phys. Rev. E 97, 012140 (2018).

[12] A. Altland and F. Haake, Equilibration and macroscopic quantum fluctuations in the Dicke model, New J. Phys. 14, 073011 (2012).

[13] A. Altland and F. Haake, Quantum Chaos and Effective Thermalization, Phys. Rev. Lett. 108, 073601 (2012).

[14] M. V. Berry, Regular and irregular semiclassical wavefunctions, J. Phys. A 10, 2083 (1977).

[15] M. V. Berry, Quantizing a classically ergodic system: Sinai's billiard and the KKR method, Ann. Phys. (NY) 131, 163 (1981).

[16] O. Bohigas, M. J. Giannoni, and C. Schmit, Characterization of Chaotic Quantum Spectra and Universality of Level Fluctuation Laws, Phys. Rev. Lett. 52, 1 (1984).

[17] R. H. Dicke, Coherence in spontaneous radiation processes, Phys. Rev. 93, 99 (1954).
[18] B. M. Garraway, The Dicke model in quantum optics: Dicke model revisited, Philos. Trans. R. Soc. Lond. Ser. A Math. Phys. Eng. Sci. 369, 1137 (2011).

[19] D. Schneble, Y. Torii, M. Boyd, E. W. Streed, D. E. Pritchard, and W. Ketterle, The onset of matter-wave amplification in a superradiant Bose-Einstein condensate, Science 300, 475 (2003).

[20] A. Blais, R.-S. Huang, A. Wallraff, S. M. Girvin, and R. J. Schoelkopf, Cavity quantum electrodynamics for superconducting electrical circuits: An architecture for quantum computation, Phys. Rev. A 69, 062320 (2004).

[21] M. Scheibner, T. Schmidt, L. Worschech, A. Forchel, G. Bacher, T. Passow, and D. Hommel, Superradiance of quantum dots, Nat. Phys. 3, 106 (2007).

[22] J. M. Fink, R. Bianchetti, M. Baur, M. Göppl, L. Steffen, S. Filipp, P. J. Leek, A. Blais, and A. Wallraff, Dressed Collective Qubit States and the Tavis-Cummings Model in Circuit Qed, Phys. Rev. Lett. 103, 083601 (2009).

[23] K. Baumann, C. Guerlin, F. Brennecke, and T. Esslinger, Dicke quantum phase transition with a superfluid gas in an optical cavity, Nature (London) 464, 1301 (2010).

[24] D. Nagy, G. Kónya, G. Szirmai, and P. Domokos, Dicke-Model Phase Transition in the Quantum Motion of a Bose-Einstein Condensate in an Optical Cavity, Phys. Rev. Lett. 104, 130401 (2010).

[25] T. Niemczyk, F. Deppe, H. Huebl, E. P. Menzel, F. Hocke, M. J. Schwarz, J. J. Garcia-Ripoll, D. Zueco, T. Hümmer, E. Solano, A. Marx, and R. Gross, Circuit quantum electrodynamics in the ultrastrong-coupling regime, Nat. Phys. 6, 772 (2010).

[26] J. Casanova, G. Romero, I. Lizuain, J. J. García-Ripoll, and E. Solano, Deep Strong Coupling Regime of the JaynesCummings Model, Phys. Rev. Lett. 105, 263603 (2010).

[27] K. Baumann, R. Mottl, F. Brennecke, and T. Esslinger, Exploring Symmetry Breaking at the Dicke Quantum Phase Transition, Phys. Rev. Lett. 107, 140402 (2011).

[28] A. Mezzacapo, U. Las Heras, J. S. Pedernales, L. DiCarlo, E. Solano, and L. Lamata, Digital quantum Rabi and Dicke models in superconducting circuits, Sci. Rep. 4, 7482 (2014).

[29] J. Cohn, A. Safavi-Naini, R. J. Lewis-Swan, J. G. Bohnet, M. Garttner, K. A. Gilmore, J. E. Jordan, A. M. Rey, J. J. Bollinger, and J. K. Freericks, Bang-bang shortcut to adiabaticity in the Dicke model as realized in a Penning trap experiment, New J. Phys. 20, 055013 (2018). 
[30] A. Safavi-Naini, R. J. Lewis-Swan, J. G. Bohnet, M. Gärttner, K. A. Gilmore, J. E. Jordan, J. Cohn, J. K. Freericks, A. M. Rey, and J. J. Bollinger, Verification of a Many-Ion Simulator of the Dicke Model Through Slow Quenches Across a Phase Transition, Phys. Rev. Lett. 121, 040503 (2018).

[31] R. J. Lewis-Swan, A. Safavi-Naini, J. J. Bollinger, and A. M. Rey, Unifying scrambling, thermalization and entanglement through measurement of fidelity out-of-time-order correlators in the Dicke model, Nat. Commun. 10, 1581 (2019).

[32] C. Emary and T. Brandes, Quantum Chaos Triggered by Precursors of a Quantum Phase Transition: The Dicke Model, Phys. Rev. Lett. 90, 044101 (2003).

[33] C. Emary and T. Brandes, Chaos and the quantum phase transition in the Dicke model, Phys. Rev. E 67, 066203 (2003).

[34] O. Castaños, R. López-Peña, E. Nahmad-Achar, J. G. Hirsch, E. López-Moreno, and J. E. Vitela, Coherent state description of the ground state in the Tavis-Cummings model and its quantum phase transitions, Phys. Scr. 79, 065405 (2009).

[35] P. Cejnar, M. Macek, S. Heinze, J. Jolie, and J. Dobes, Monodromy and excited-state quantum phase transitions in integrable systems: Collective vibrations of nuclei, J. Phys. A 39, L515 (2006).

[36] P. Pérez-Fernández, A. Relaño, J. M. Arias, P. Cejnar, J. Dukelsky, and J. E. García-Ramos, Excited-state phase transition and onset of chaos in quantum optical models, Phys. Rev. E 83, 046208 (2011).

[37] T. Brandes, Excited-state quantum phase transitions in Dicke superradiance models, Phys. Rev. E 88, 032133 (2013).

[38] M. A. Bastarrachea-Magnani, S. Lerma-Hernández, and J. G. Hirsch, Comparative quantum and semiclassical analysis of atom-field systems. I. Density of states and excited-state quantum phase transitions, Phys. Rev. A 89, 032101 (2014).

[39] J. P. J. Rodriguez, S. A. Chilingaryan, and B. M. RodríguezLara, Critical phenomena in an extended Dicke model, Phys. Rev. A 98, 043805 (2018).

[40] M. A. Bastarrachea-Magnani, S. Lerma-Hernández, and J. G. Hirsch, Comparative quantum and semiclassical analysis of atom-field systems. II. Chaos and regularity, Phys. Rev. A 89, 032102 (2014).

[41] M. A. Bastarrachea-Magnani, B. L. del Carpio, S. LermaHernández, and J. G. Hirsch, Chaos in the Dicke model: Quantum and semiclassical analysis, Phys. Scr. 90, 068015 (2015).

[42] J. Chávez-Carlos, M. A. Bastarrachea-Magnani, S. LermaHernández, and J. G. Hirsch, Classical chaos in atom-field systems, Phys. Rev. E 94, 022209 (2016).

[43] M. A. Bastarrachea-Magnani, B. López-del-Carpio, J. ChávezCarlos, S. Lerma-Hernández, and J. G. Hirsch, Delocalization and quantum chaos in atom-field systems, Phys. Rev. E 93, 022215 (2016).

[44] J. Chávez-Carlos, B. López-del-Carpio, M. A. BastarracheaMagnani, P. Stránský, S. Lerma-Hernández, L. F. Santos, and J. G. Hirsch, Quantum and Classical Lyapunov Exponents in Atom-Field Interaction Systems, Phys. Rev. Lett. 122, 024101 (2019).

[45] P. Stránsk, M. Macek, and P. Cejnar, Excited-state quantum phase transitions in systems with two degrees of freedom: Level density, level dynamics, thermal properties, Ann. Phys. (NY) 345, 73 (2014).
[46] M. Caprio, P. Cejnar, and F. Iachello, Excited state quantum phase transitions in many-body systems, Ann. Phys. (NY) 323, 1106 (2008).

[47] M. de Aguiar, K. Furuya, C. Lewenkopf, and M. Nemes, Chaos in a spin-boson system: Classical analysis, Ann. Phys. (NY) 216, 291 (1992).

[48] Y. A. Kuznetsov, Elements of Applied Bifurcation Theory (Springer Science \& Business Media, New York, 2013), Vol. 112.

[49] A. Andronov, E. Leontovich, I. Gordon, and A. Maier, Theory of Bifurcations of Dynamic Systems on a Plane (Israel Program for Scientific Translations, New York, 1971).

[50] V. I. Arnol'd, Geometrical Methods in the Theory of Ordinary Differential Equations (Springer Science \& Business Media, New York, 2012), Vol. 250.

[51] R. de J. León-Montiel, J. Svozilík, and J. P. Torres, Generation of a tunable environment for electrical oscillator systems, Phys. Rev. E 90, 012108 (2014).

[52] R. de J. León-Montiel, M. A. Quiroz-Juárez, R. QuinteroTorres, J. L. Domínguez-Juárez, H. M. Moya-Cessa, J. P. Torres, and J. L. Aragón, Noise-assisted energy transport in electrical oscillator networks with off-diagonal dynamical disorder, Sci. Rep. 5, 17339 (2015).

[53] M. Quiroz-Juárez, J. Aragon, R. de J. León-Montiel, R. Vazquez-Medina, J. Dominguez-Juarez, and R. QuinteroTorres, Emergence of a negative resistance in noisy coupled linear oscillators, Europhys. Lett. 116, 50004 (2017).

[54] R. de J. León-Montiel, M. A. Quiroz-Juárez, J. L. DomínguezJuárez, R. Quintero-Torres, J. L. Aragón, A. K. Harter, and Y. N. Joglekar, Observation of slowly decaying eigenmodes without exceptional points in floquet dissipative synthetic circuits, Commun. Phys. 1, 88 (2018).

[55] F. Yoshihara, T. Fuse, S. Ashhab, K. Kakuyanagi, S. Saito, and K. Semba, Superconducting qubit-oscillator circuit beyond the ultrastrong-coupling regime, Nat. Phys. 13, 44 (2017).

[56] Y. Zhang, L. Yu, J.-Q. Liang, G. Chen, S. Jia, and F. Nori, Quantum phases in circuit QED with a superconducting qubit array, Sci. Rep. 4, 4083 (2014).

[57] A. Wallraff, D. I. Schuster, A. Blais, L. Frunzio, R.-S. Huang, J. Majer, S. Kumar, S. M. Girvin, and R. J. Schoelkopf, Strong coupling of a single photon to a superconducting qubit using circuit quantum electrodynamics, Nature (London) 431, 162 (2004).

[58] E. Nahmad-Achar, O. Castaños, R. López-Peña, and J. G. Hirsch, Mathematical methods in quantum optics: The Dicke model, Phys. Scr. 87, 038114 (2013).

[59] M. A. Lyapunov, The General Problem of the Stability of Motion (in Russian) (Kharkov Mathematical Society, Moscow, 1892).

[60] V. I. Oseledets, A multiplicative ergodic theorem. characteristic Lyapunov, exponents of dynamical systems, Tr. Mosk. Mat. Obs. 19, 179 (1968).

[61] G. Benettin, L. Galgani, A. Giorgilli, and J.-M. Strelcyn, Lyapunov characteristic exponents for smooth dynamical systems and for Hamiltonian systems; a method for computing all of them. Part 1: Theory, Meccanica 15, 9 (1980).

[62] M. T. Rosenstein, J. J. Collins, and C. J. De Luca, A practical method for calculating largest Lyapunov exponents from small data sets, Physica D 65, 117 (1993). 
[63] M. J. Steel and M. J. Collett, Quantum state of two trapped Bose-Einstein condensates with a Josephson coupling, Phys. Rev. A 57, 2920 (1998).

[64] A. Polkovnikov, Phase space representation of quantum dynamics, Ann. Phys. (NY) 325, 1790 (2010).

[65] J. Schachenmayer, A. Pikovski, and A. M. Rey, ManyBody Quantum Spin Dynamics with Monte Carlo Trajectories on a Discrete Phase Space, Phys. Rev. X 5, 011022 (2015).

[66] R. F. Fox and T. C. Elston, Chaos and a quantum-classical correspondence in the kicked top, Phys. Rev. E 50, 2553 (1994).
[67] P. Stránsk, M. Macek, A. Leviatan, and P. Cejnar, Excitedstate quantum phase transitions in systems with two degrees of freedom: II. Finite-size effects, Ann. Phys. (NY) 356, 57 (2015).

[68] R. Vazquez-Medina, O. Jimenez-Ramirez, M. QuirozJuarez, and J. Aragon, Arbitrary waveform generator biologically inspired, Chaos, Solitons Fractals 51, 36 (2013).

[69] S. Smith, Digital Signal Processing: A Practical Guide for Engineers and Scientists (Elsevier, New York, 2013). 